\title{
Investigation of a Potential Pharmacokinetic Interaction Between Nebivolol and Fluvoxamine in Healthy Volunteers
}

\begin{abstract}
Ana-Maria Gheldiu ${ }^{1}$, Laurian Vlase ${ }^{1}$, Adina Popa ${ }^{2}$, Corina Briciu², Dana Muntean ${ }^{1}$, Corina Bocsan ${ }^{3}$, Anca Buzoianu ${ }^{3}$, Marcela Achim ${ }^{1}$, Ioan Tomuta ${ }^{1}$, Ioana Todor ${ }^{1}$, Daniel Leucuta ${ }^{4}$, Maria Neag ${ }^{3}$

Iuliu Hatieganu University of Medicine and Pharmacy, Faculty of Pharmacy, Cluj-Napoca, Romania; ${ }^{1}$ Department of Pharmaceutical Technology and Biopharmaceutics, ${ }^{2}$ Department of Clinical Pharmacy, ${ }^{3}$ Department of Pharmacology, Toxicology and Clinical Pharmacology, and ${ }^{4}$ Department of Medical Informatics and Biostatistics
\end{abstract}

Received, January 20, 2017; Accepted, April 6, 2017; April 7, 2017.

\begin{abstract}
PURPOSE: To investigate whether fluvoxamine coadministration can influence the pharmacokinetic properties of nebivolol and its active hydroxylated metabolite (4-OH-nebivolol) and to assess the consequences of this potential pharmacokinetic interaction upon nebivolol pharmacodynamics. METHODS: This open-label, non-randomized, sequential clinical trial consisted of two periods: Period 1 (Reference), during which each volunteer received a single dose of $5 \mathrm{mg}$ nebivolol and Period 2 (Test), when a combination of $5 \mathrm{mg}$ nebivolol and $100 \mathrm{mg}$ fluvoxamine was given to all subjects, after a 6-days pretreatment regimen with fluvoxamine (50-100 mg/day). Non-compartmental analysis was used to determine the pharmacokinetic parameters of nebivolol and its active metabolite. The pharmacodynamic parameters (blood pressure and heart rate) were assessed at rest after each nebivolol intake, during both study periods. RESULTS: Fluvoxamine pretreatment increased $\mathrm{C}_{\max }$ and $\mathrm{AUC}_{0-\infty}$ of nebivolol $\left(\mathrm{C}_{\max }: 1.67 \pm 0.690\right.$ vs $2.20 \pm 0.970 \mathrm{ng} / \mathrm{mL} ; \mathrm{AUC}_{0-\infty}: 12.1$ \pm 11.0 vs $19.3 \pm 19.5 \mathrm{ng} * \mathrm{~h} / \mathrm{mL})$ and of its active metabolite $\left(\mathrm{C}_{\max }: 0.680 \pm 0.220\right.$ vs $0.960 \pm 0.290 \mathrm{ng} / \mathrm{mL}$; $\mathrm{AUC}_{0-\infty}: 17.6 \pm 20.1$ vs $25.5 \pm 29.9 \mathrm{ng} * \mathrm{~h} / \mathrm{mL}$ ). Apart from $\mathrm{C}_{\max }, \mathrm{AUC}_{0-\mathrm{t}}$ and $\mathrm{AUC}_{0-\infty}$, the other pharmacokinetic parameters $\left(t_{\max }, k_{e l}\right.$ and $\left.t_{1 / 2}\right)$ were not significantly different between study periods. As for the pharmacodynamic analysis, decreases in blood pressure and heart rate after nebivolol administration were similar with and without fluvoxamine concomitant intake. CONCLUSIONS: Due to enzymatic inhibition, fluvoxamine increases the exposure to nebivolol and its active hydroxylated metabolite in healthy volunteers. This did not influence the blood pressure and heart-rate lowering effects of the beta-blocker administered as single-dose. However, more detail studies involving actual patients are required to further investigate the clinical relevance of this drug interaction.
\end{abstract}

This article is open to POST-PUBLICATION REVIEW. Registered readers (see "For Readers") may comment by clicking on ABSTRACT on the issue's contents page.

\section{INTRODUCTION}

Nebivolol is a highly lipophilic, third-generation beta-blocker used for the treatment of hypertension and chronic heart failure (HF) $(1,2)$. Until now, it was proved to possess the highest selectivity for the $\beta 1$-adrenergic receptors (up to 320 -fold vs $\beta 2$ ) among the beta-blockers. With a unique hemodynamic profile, this drug exhibits a vasodilatory role by interacting with the endothelial L-arginine/NO pathway and thus enhancing NO release and bioavailability $(3,4)$. In addition, nebivolol exerts an antioxidant effect which may also contribute to its vasodilatory action (5). This beta-blocker is available as a racemate of $d$ nebivolol and 1-nebivolol, two enantiomers that act synergistically in order to achieve blood pressure (BP) reduction. The selective blocking of $\beta 1$ - receptors is determined almost exclusively by $\mathrm{d}$ nebivolol, while 1-nebivolol mainly provides the vasodilatory effect $(6,7)$.

Nebivolol is rapidly absorbed after oral administration and food intake has a minimal impact on this process $(2,4)$. According to a study performed on rats, the low bioavailability of nebivolol is due to first-pass metabolism through the gut (8). In addition, it was also revealed that this beta-blocker is subjected to hepatic metabolism primarily through CYP2D6 (9). Nebivolol is extensively metabolized by oxidation,

Corresponding Author: Laurian Vlase, Iuliu Hatieganu University of Medicine and Pharmacy, Faculty of Pharmacy, Department of Pharmaceutical Technology and Biopharmaceutics, Victor Babes 41, Cluj-Napoca, Romania, email: vlaselaur@yahoo.com; laurian.vlase@umfcluj.ro 
glucuronidation, hydroxylation and N-dealkylation, each metabolic pathway providing different metabolites (10,11). The hydroxylated and glucuronidated metabolites are considered pharmacologically active, which contributes to an equally similar clinical profile for both CYP2D6 phenotypes $(12,13)$. As CYP2D6 is a polymorphic isoenzyme, extensive metabolizers (EMs) and poor metabolizers (PMs) exhibit different pharmacokinetic properties. Oral bioavailability varies between $12 \%$ in EMs versus $96 \%$ in PMs (10) and studies revealed that the elimination halflife $\left(t_{1} / 2\right)$ of nebivolol and its hydroxyl-metabolites were increased 3-5 fold, respectively 2 -fold in the PM group (14).

Fluvoxamine is a selective serotonin reuptake inhibitor (SSRI) widely used for the treatment of major depressive episodes, obsessive-compulsive disorder, social anxiety disorder and bulimia nervosa. Almost completely absorbed after oral administration, its peak plasma concentrations $\left(\mathrm{C}_{\max }\right)$ is reached in 3 to 8 hours, the mean $t_{1 / 2}$ is about 15 hours and steady-state plasma levels are obtained after approximately 1 week $(15,16)$. With a nonlinear pharmacokinetics, fluvoxamine undergoes hepatic biotransformation through oxidative demethylation and oxidative deamination resulting in inactive metabolites (17). Most importantly, a great concern lies in the potential interactions of fluvoxamine, as it is known to be a potent inhibitor of CYP1A2 and CYP2C19, a moderate inhibitor of CYP2C9 and CYP3A4, and a weak inhibitor of CYP2D6 (18).

Depressive disorder was found to be highly prevalent in patients with cardiovascular diseases (19). For example, a meta-analysis reported a prevalence of $21.5 \%$ for depression in patients with $\mathrm{HF}$ and concluded that this comorbidity was present in at least 1 out of 5 patients (20). Considering this epidemiological context, the present study will offer valuable information regarding the safety profile of nebivolol. Also, the results could help clinicians choose appropriate combination strategies when pharmacological treatment for cardiovascular disease and depressive disorder is equally needed. Therefore, although fluvoxamine is only a weak inhibitor of CYP2D6, it is imperative to investigate whether it can influence the pharmacokinetics of cardiovascular drugs known to be CYP2D6 substrates, like nebivolol. The main objective of the present research was to verify whether a pharmacokinetic interaction between nebivolol and fluvoxamine does exist and if so, to provide a first insight concerning its potential clinical relevance.

\section{METHODS}

\section{Volunteers}

Inclusion criteria required subjects to be healthy, nonsmoking Caucasian males or females, 18 to 55 years old. Their health status was assessed based on medical history, physical examination, vital signs assessment (blood pressure (BP) and heart rate (HR)), electrocardiogram (ECG) and clinical laboratory tests (hematology, blood chemistry, serology and urinalysis). Volunteers were excluded from the study if they had a history of drug allergy, alcohol or drug abuse, any clinically relevant abnormality identified at the physical examination, including resting HR less than 60 beats per minute and BP less than $90 / 50 \mathrm{mmHg}$. Those who used any medication within 14 days before the trial, who donated blood within 90 days before the study initiation or had a significant medical history that can alter drug response or a lifestyle incompatibility with the study requirements, were also considered ineligible subjects for the present research.

The clinical protocol was reviewed and approved by the Ethics Committee of the University of Medicine and Pharmacy "Iuliu Hatieganu", ClujNapoca, Romania. All volunteers gave their written informed consent prior to study inclusion. The research was conducted according to the Declaration of Helsinki and its amendments and Good Clinical Practice guidelines.

\section{Study design}

The study was designed as an open-label, nonrandomized, sequential study, that consisted of 2 periods. Day 1 of clinical trial (Reference), each volunteer received a single dose of $5 \mathrm{mg}$ nebivolol. In between the Reference and Test periods of the study, the subjects were treated with a single daily dose of $50 \mathrm{mg}$ fluvoxamine for 3 days (day 2, 3 and 4 of clinical trial), followed by 3 days in which they were given $100 \mathrm{mg}$ fluvoxamine as a single daily dose (day 5,6 and 7). This pretreatment, with the enzymatic inhibitor (fluvoxamine), was done in order to create a repeated dosing regimen of fluvoxamine plasma concentrations. Day 8 of clinical trial (Test), all volunteers received a single dose of $5 \mathrm{mg}$ nebivolol + a single dose of $100 \mathrm{mg}$ fluvoxamine. All volunteers were required to undergo a 12-hour overnight fast prior to drug administration and all drugs were administered in the morning, with at least $150 \mathrm{~mL}$ water. The volunteers were quarantined in a controlled environment, at the clinical research unit, on day 1 and day 8 of the study, where they remained for 12 
hours after drug administration. Standardized meals were provided for breakfast, lunch and dinner at 3,6 and 10 hours after dosing. Water intake was prohibited 1 hour before and two hours after drug administration. Also, drugs (other than the study medication and oral contraceptives, if needed), alcoholic or caffeine-containing beverages and smoking were not allowed.

The pharmaceutical products used were Nebilet for nebivolol (5 mg tablets, manufactured by BerlinChemie AG (Menarini Group), Germany) and Fevarin for fluvoxamine (50 and $100 \mathrm{mg}$ coated tablets, manufactured by Abbott Healthcare Products B.V, The Netherlands ).

\section{Blood plasma samples collection and analytical method description}

Venous blood $(5 \mathrm{ml})$ was drawn into heparinized tubes, on the first and last day of study, at pre-dose (0 hours), as well as at 1/2,1,1 1/2, 2, $21 / 2,3,4,6$, $8,10,12,24,36$ and 48 hours after drug administration . The separated plasma was frozen ($20^{\circ} \mathrm{C}$ ) until analysis.

The plasma concentrations of nebivolol and its active hydroxylated metabolite (4-OH-nebivolol) were determined using validated high-performance liquid chromatography (HPLC)-tandem mass spectroscopy methods (MS). The analytical method used was not enantioselective. The HPLC system, an Agilent 1100 series (binary pump, autosampler, thermostat) (Agilent Technologies, USA) was coupled with a Brucker Ion Trap SL (Brucker Daltonics GmbH, Germany). A Zorbax SB-C18 chromatographic column (100 $\mathrm{mm}$ x $3.0 \mathrm{~mm}$ i.d., 3.5 $\mu \mathrm{m})$ (Agilent Technologies) was used and the mobile phase consisted of $64: 36(\mathrm{v} / \mathrm{v}) \quad 0.2 \%(\mathrm{~V} / \mathrm{V})$ formic acid in water: methanol. The thermostat temperature was set at $45{ }^{\circ} \mathrm{C}$, while the flow rate was $1 \mathrm{ml} / \mathrm{min}$. In these chromatographic conditions, the retention times for nebivolol and its metabolite were $5.0 \mathrm{~min}$ and $6.4 \mathrm{~min}$, respectively. The mass spectrometry detection was in positive-ion electrospray mode, multiple-reaction monitoring. The monitored ion transitions were m/z 406 for nebivolol and m/z 404 from $\mathrm{m} / \mathrm{z} 422$ for its hydroxylated metabolite.

The preparation of each sample was performed in an Eppendorf tube, in which $0.1 \mathrm{ml}$ perchloric acid $7 \%(\mathrm{~V} / \mathrm{V})$ in water was added to $0.2 \mathrm{ml}$ plasma. The tube was vortex-mixed for 10 seconds, then centrifuged for $6 \mathrm{~min}$ at $9000 \mathrm{rpm}$. The supernatant was transferred to an autosampler vial and $40 \mu \mathrm{l}$ were injected into the chromatographic system. The analytical method was validated in terms of specificity, linearity, intra- and inter-day precision, accuracy and analyte recovery. The calibration curves of nebivolol and its metabolite were linear at a concentration range of $0.1-16 \mathrm{ng} / \mathrm{ml}$ plasma, with correlation coefficients (r) $0.9925 \pm 0.0023$ (mean \pm standard deviation $(\mathrm{SD}), \mathrm{n}=5$ ) for nebivolol and $0.9918 \pm 0.0031$ for $4-\mathrm{OH}-$ nebivolol, respectively. For nebivolol, intra- and inter-day precision was less than $12.3 \%$, the accuracy (bias) less than $6.31 \%$ and the recovery ranged between $86-105 \%$, respectively. For 4-OH-nebivolol, intra- and inter-day precision was less than $9.2 \%$, the accuracy less than $-8.8 \%$ and the recovery ranged between $93-110 \%$, respectively.

\section{Pharmacokinetic analysis}

The pharmacokinetic (PK) parameters of nebivolol and its active metabolite (4-0H-nebivolol), when given alone and in combination with fluvoxamine, were determined by a non-compartmental analysis using Phoenix WinNonlin Professional software (Version 6.3, Pharsight Corp., Mountain View, CA, USA). Peak plasma concentration $\left(\mathrm{C}_{\max }, \mathrm{ng} / \mathrm{ml}\right)$ and the time to reach $\mathrm{C}_{\max }\left(\mathrm{t}_{\max }, \mathrm{h}\right)$ were noted directly from the experimental data, respectively from the concentration vs. time profiles of both analytes. The area under the time-concentration curve from time 0 to the last measurable time $\left(\mathrm{AUC}_{0-\mathrm{t}}\right)$ was calculated using the trapezoidal rule-extrapolation method, while the area under the time-concentration curve extrapolated to infinity $\left(\mathrm{AUC}_{0-\infty}\right)$ was obtained as follows: $\mathrm{AUC}_{0-\mathrm{t}}+\mathrm{C}_{\mathrm{t}} / \mathrm{k}_{\mathrm{el}}\left(\mathrm{C}_{\mathrm{t}}\right.$ - the last quantifiable drug concentration, $\mathrm{k}_{\mathrm{el}}$ is the elimination rate constant). $\mathrm{k}_{\mathrm{el}}$ was calculated by linear regression analysis of the terminal phase of the log concentration-time profile and the half-life $\left(\mathrm{t}_{1 / 2}\right)$ was obtained using the following formula $t_{1 / 2}=0.693 / \mathrm{k}_{\mathrm{el}}$.

\section{Phenotype analysis}

Potential PMs were identified based on the $\mathrm{AUC}_{0-\infty}$ metabolic ratio $\left(\mathrm{AUC}_{0-\infty}\right.$ nebivolol/ $/ \mathrm{AUC}_{0-\infty} 4-\mathrm{OH}-$ nebivolol) corresponding to the Reference period. This was performed for each volunteer and subsequently, the AUC metabolic ratios were evaluated for standard normal distribution and outliers to determine phenotypic distribution. The higher values of AUC metabolic ratio (nebivolol/4$\mathrm{OH}$-nebivolol) were associated with the PM status, while the rest of the values were considered to represent the EM group. Considering that the aim of this research was not to investigate the influence of each phenotypic group upon nebivolol pharmacokinetics, but to assess the existence of a potential pharmacokinetic drug-drug interaction between nebivolol and fluvoxamine, PMs were 
excluded from the study and the final analysis included only the results attributed to the EM group.

\section{Pharmacodynamic analysis}

The pharmacodynamic (PD) parameters, respectively systolic/diastolic blood pressure (SBP/DBP) and HR, were measured and recorded during both study periods (day 1 and day 8) while the volunteers were at rest, in a supine position, before and at 1, 2, 3, 4, 5, 6, 7, 8, 9, 10, 11, 12, 24, 36 and 48 hours after nebivolol intake. A semiautomatic blood pressure monitor (BP A50, MicroLife, Switzerland) was used to obtain the required measurements and for each time-point mentioned before, two assessments of BP and HR, at 2 minutes apart, were performed, with the mean values being included in the statistical analysis. After obtaining the PD parameters, the change of each measurement (SBP, DBP and HR) in the absence or presence of drug interaction was evaluated. More precisely, the effect of nebivolol on resting $\mathrm{SBP} / \mathrm{DBP}$ and $\mathrm{HR}$ at each measuring point before and after fluvoxamine concomitant administration, was calculated as the percentage change from baseline (before nebivolol intake). Afterwards, the parameters corresponding to the two study periods were compared by using a graphical and also a statistical approach (Test versus Reference).

\section{STATISTICAL ANALYSIS}

The statistical analysis selected to test for differences between study periods for all the PD parameters was a general linear model. The differences between study periods for normally distributed PK parameters were tested with a paired t-test, while a Wilcoxon signed-ranks test was used for those who didn't follow a normal distribution.

The sample size for the main outcome of the study - the differences between PK parameters $\left(\mathrm{C}_{\max }\right.$ and AUC) for the two study periods, were computed for a level of significance of 0.05 , a power of $90 \%$, a two tailed $p$-value paired t-test using $\mathrm{G}^{*}$ Power (Version 3.0.10, Germany) (21). We used the means and standard deviations from articles comparing the same $5 \mathrm{mg}$ dose of nebivolol and nebivolol with another inhibitor - paroxetine (22) and cimetidine (23), and we simulated all predictions for a correlation coefficient between the two periods (0.01 -0.99 ) and 0.5 . We obtained a range of predictions between 3 to 19 subjects, and we finally chose a target sample size of 20 .
The statistical analysis was performed using Phoenix WinNonlin Professional software (Version 6.3, Pharsight Corp., Mountain View, CA, USA) and $\mathrm{R}$ environment for statistical computing and graphics (Version 3.2.3, R Core Team, Vienna, Austria). A two tailed $p$ value less than 0.05 was considered to be statistically significant.

The bioequivalence assessment methodology was used in order to detect significant changes in PK parameters and to evaluate a possible clinical relevance associated with the potential drug interaction between nebivolol and fluvoxamine. For this reason, Schuirmann's two one-sided t tests procedure was engaged to determine $90 \%$ confidence intervals (CI) of the Test/Reference period ratios for $\mathrm{C}_{\max }, \mathrm{AUC}_{0-\mathrm{t}}$ and $\mathrm{AUC}_{0-\infty}$ (log transformed). Bioequivalence between nebivolol administered alone or in combination with fluvoxamine was assumed when the ratio Test/Reference fell within the $90 \%$ CI $0.80-1.25$ reference range. For $t_{\max }$, the bioequivalence range was expressed as untransformed data and significance was tested by using the nonparametric Friedman test.

\section{RESULTS}

\section{Phenotype analysis}

The results of the $\mathrm{AUC}_{0-\infty}$ metabolic ratio (nebivolol/4-OH-nebivolol) assessment showed that two subjects could be considered as having a PM status (subject number 5 and 16). For the PM group, the mean $\mathrm{AUC}_{0-\infty}$ metabolic ratio was $7.96 \pm 1.65$ $\left(\mathrm{AUC}_{0-\infty}\right.$ nebivolol: $236 \pm 4.98 \mathrm{ng} * \mathrm{~h} / \mathrm{mL} ; \mathrm{AUC}_{0-\infty} 4-$ OH-nebivolol: $30.2 \pm 5.64 \mathrm{ng} * \mathrm{~h} / \mathrm{mL}$ ), whereas for the EM group the mean value was very different, respectively $1.05 \pm 0.697 \quad\left(\mathrm{AUC}_{0-\infty}\right.$ nebivolol: 12.1 $\pm 11.0 \quad \mathrm{ng} * \mathrm{~h} / \mathrm{mL} ; \quad \mathrm{AUC}_{0-\infty} \quad$ 4-OH-nebivolol: $17.6 \pm 20.1 \mathrm{ng}^{*} \mathrm{~h} / \mathrm{mL}$ ). According to study protocol, in order to avoid any interference with the outcome of this assay, the potential PMs were excluded from the investigation and only data pertaining to the remaining 18 subjects (EMs) was included in the final PK, PD and statistical evaluation.

\section{Demographics}

Demographic data of the EM group that was included in the final analysis are presented in Table 1. All volunteers completed the study without protocol deviations. 


\section{Pharmacokinetics}

Mean plasma concentration-time profiles of nebivolol and its corresponding hydroxylated active metabolite, when administered alone or in combination with fluvoxamine, are presented in Figure 1 (nebivolol) and Figure 2 (nebivolol 4-OHnebivolol).
Table 1. Demographics of the subjects included in the final analysis (EMs)

\begin{tabular}{ll}
\hline Characteristic & Value \\
Number of subjects & 18 \\
Gender (number) & \\
$\quad$ Women & 8 \\
$\quad$ Men & 10 \\
Age (years, mean $\left.\pm \mathrm{SD}^{*}\right)$ & $24.83 \pm 3.16$ \\
$\mathrm{BMI} *\left(\mathrm{~kg} / \mathrm{m}^{2}\right.$, mean $\left.\pm \mathrm{SD}^{*}\right)$ & $22.30 \pm 2.57$ \\
\hline *SD - standard deviation; **BMI - body mass index
\end{tabular}

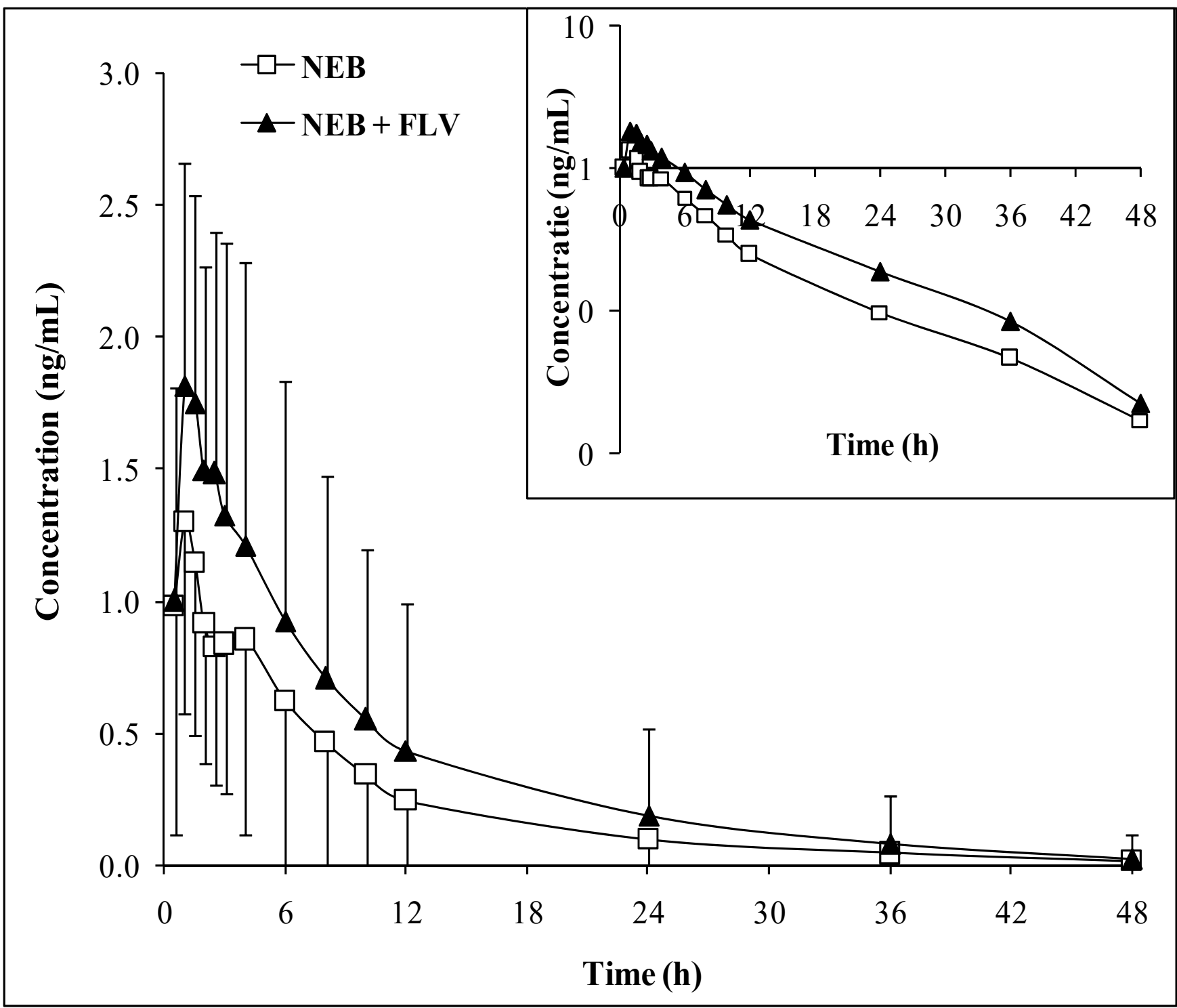

Figure 1. Mean \pm standard deviation (SD) plasma concentration-time curves of nebivolol (NEB; $5 \mathrm{mg}$, p.o.) administered alone or in combination with fluvoxamine (FLV; $100 \mathrm{mg}$, p.o.) after 6 days pretreatment with FLV (50 mg/day for 3 days and $100 \mathrm{mg}$ /day for another 3 days), $\mathrm{n}=18$ (extensive metabolizers - EMs). Insert: semi-logarithmic presentation. 


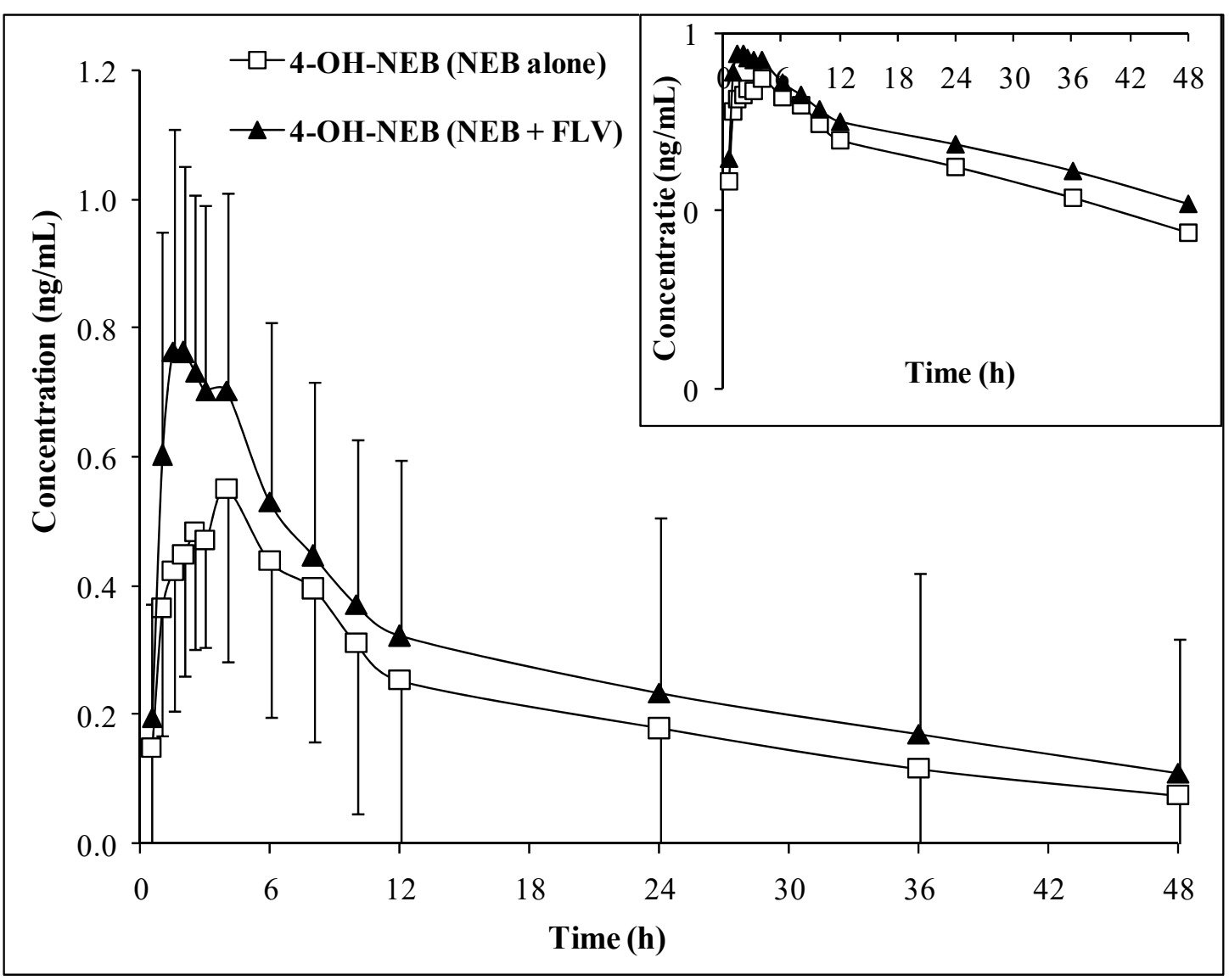

Figure 2. Mean \pm standard deviation (SD) plasma concentration-time curves of 4-OH - nebivolol (4-OH-NEB; nebivolol hydroxylated active metabolite) corresponding to nebivolol (NEB; $5 \mathrm{mg}$, p.o.) administered alone or in combination with fluvoxamine (FLV; $100 \mathrm{mg}$, p.o.), after 6 days pretreatment with FLV (50 mg/day for 3 days and $100 \mathrm{mg} /$ day for another 3 days), $\mathrm{n}=18$ (extensive metabolizers - EMs). Insert: semi-logarithmic presentation.

Following the graphical representation of plasma concentration profiles for nebivolol and its active metabolite, the mean pharmacokinetic parameters for each analyte, when administered alone or in combination with fluvoxamine, as well as the statistical test results are presented in Table 2 (nebivolol) and Table 3 (4-OHnebivolol).

Table 2. Summary of pharmacokinetic (PK) parameters and statistical comparison of nebivolol (NEB) PK parameters, following administration of $5 \mathrm{mg}$ nebivolol given as a single dose, with or without fluvoxamine (FLV; multiple-dose pretreatment, $50-100 \mathrm{mg} / \mathrm{day}$ ), in 18 healthy volunteers (extensive metabolizers - EMs).

\begin{tabular}{llll}
\hline PK parameters & NEB & NEB + FLV & $\boldsymbol{p}^{*}$ value \\
\hline $\mathrm{C}_{\max }(\mathrm{ng} / \mathrm{mL})$ & $1.67 \pm 0.69$ & $2.20 \pm 0.97$ & $0.029, \mathbf{S}$ \\
& $1.52(1.16-2.19)$ & $2.21(1.37-2.62)$ & \\
$\mathrm{t}_{\max }(\mathrm{h})$ & $1.81 \pm 1.19$ & $1.72 \pm 1.00$ & $0.924, \mathbf{N S}$ \\
& $1.5(1-2.38)$ & $1.25(1-2.38)$ & \\
$\mathrm{AUC}_{0-\mathrm{t}}(\mathrm{ng} * \mathrm{~h} / \mathrm{mL})$ & $10.4 \pm 10.5$ & $16.3 \pm 18.1$ & $0.002, \mathbf{S}$ \\
& $7.87(4.88-14.83)$ & $11.89(7.2-20.82)$ & \\
$\mathrm{AUC}_{0-\infty}(\mathrm{ng} * \mathrm{~h} / \mathrm{mL})$ & $12.1 \pm 11.0$ & $19.3 \pm 19.5$ & $0.002, \mathbf{S}$ \\
& $7.87(4.88-14.83)$ & $11.89(7.2-20.82)$ & $0.338, \mathbf{N S}$ \\
$\mathrm{k}_{\mathrm{el}}(\mathrm{L} / \mathrm{h})$ & $0.13 \pm 0.09$ & $0.11 \pm 0.06$ & \\
& $0.11(0.08-0.15)$ & $0.09(0.06-0.17)$ & $0.799, \mathbf{N S}$ \\
$\mathrm{t}_{1 / 2}(\mathrm{~h})$ & $7.05 \pm 3.69$ & $9.15 \pm 6.74$ & \\
& $6.19(4.6-8.96)$ & $7.58(4.14-11.57)$ & \\
\hline
\end{tabular}

Data is presented as mean \pm 1 standard deviation (SD) and median and interquartile range; *statistically significant (S) when $p<0.05$; NS - non-significant 
Table 3. Summary of pharmacokinetic (PK) parameters and statistical comparison of 4-OH-nebivolol (4-OH-NEB; active metabolite of nebivolol) PK parameters, following administration of $5 \mathrm{mg}$ nebivolol (NEB) given as a single dose, with or without fluvoxamine (FLV; multiple-dose pretreatment, $50-100 \mathrm{mg} /$ day), in 18 healthy volunteers (extensive metabolizers EMs).

\begin{tabular}{|c|c|c|c|}
\hline PK parameters & 4-OH-NEB & 4-OH-NEB + FLV & $p^{*}$ value \\
\hline $\mathrm{C}_{\max }(\mathrm{ng} / \mathrm{mL})$ & $\begin{array}{l}0.68 \pm 0.22 \\
0.66(0.52-0.78)\end{array}$ & $\begin{array}{l}0.96 \pm 0.29 \\
0.95(0.81-1.18)\end{array}$ & $<0.001, \mathbf{S}$ \\
\hline $\mathrm{t}_{\max }(\mathrm{h})$ & $\begin{array}{l}3.00 \pm 1.65 \\
2.5(2-4)\end{array}$ & $\begin{array}{l}2.42 \pm 1.13 \\
2(1.5-3.75)\end{array}$ & $0.15, \mathbf{N S}$ \\
\hline $\mathrm{AUC}_{0-\mathrm{t}}\left(\mathrm{ng}{ }^{*} \mathrm{~h} / \mathrm{mL}\right)$ & $\begin{array}{l}9.69 \pm 9.31 \\
5.72(3-13.1)\end{array}$ & $\begin{array}{l}13.0 \pm 11.5 \\
9.69(4.4-13.98)\end{array}$ & $0.002, \mathbf{S}$ \\
\hline $\mathrm{AUC}_{0-\infty}\left(\mathrm{ng}^{*} \mathrm{~h} / \mathrm{mL}\right)$ & $\begin{array}{l}17.6 \pm 20.1 \\
8.21(4.66-21.16)\end{array}$ & $\begin{array}{l}25.5 \pm 29.9 \\
16.43(5.75-32.07)\end{array}$ & $0.010, \mathbf{S}$ \\
\hline $\mathrm{k}_{\mathrm{el}}(\mathrm{L} / \mathrm{h})$ & $\begin{array}{l}0.07 \pm 0.06 \\
0.06(0.03-0.12)\end{array}$ & $\begin{array}{l}0.10 \pm 0.13 \\
0.04(0.02-0.11)\end{array}$ & $0.670, \mathbf{N S}$ \\
\hline$t_{1 / 2}(h)$ & $\begin{array}{l}19.5 \pm 19.8 \\
12.84(5.97-26.54)\end{array}$ & $\begin{array}{l}25.9 \pm 26.4 \\
18.51(6.39-31.98)\end{array}$ & $0.442, \mathbf{N S}$ \\
\hline
\end{tabular}

Data is presented as mean \pm 1 standard deviation (SD) and median and interquartile range; *statistically significant (S) when $P<0.05$; NS - non-significant

The results of the bioequivalence evaluation that implied a comparison of the main pharmacokinetic parameters $\left(\mathrm{C}_{\max }, \mathrm{t}_{\max }, \mathrm{AUC}_{0-\mathrm{t}}\right.$ and $\left.\mathrm{AUC}_{0-\infty}\right)$ between the two study periods are shown in Table 4. The table includes the parametric $90 \%$ CI for the ratio Test/Reference period of the mean pharmacokinetic parameters $\mathrm{C}_{\max }, \quad \mathrm{AUC}_{0-\mathrm{t}}$, and $\mathrm{AUC}_{0-\infty} \quad(\log$ transformed) of nebivolol and its hydroxylated metabolite, as well as the significance of the difference for $t_{\max }$.

\section{Pharmacodynamics}

Time course of changes corresponding to the mean pharmacodynamic parameters (SBP, DBP and HR) measured after nebivolol $5 \mathrm{mg}$ single-dose intake, in the presence and absence of fluvoxamine, are depicted in Figure 3 (SBP-a, DBP-b, HR-c). Statistical analysis did not reveal significant differences between the two study periods regarding the effect induced by nebivolol upon the vital signs (data not shown).

\section{DISCUSSION}

One of the most important criteria when selecting a SSRI is the potential for involvement in pharmacokinetic drug interactions. This characteristic is linked to their potency for inhibition of cytochrome P450 isoenzymes involved in the hepatic metabolism of most of the drugs $(17,24)$. Alongside fluoxetine and paroxetine, fluvoxamine is considered one of the SSRIs with the highest risk of interaction as it influences the activity of various isoenzymes and perturbs the pharmacokinetics of numerous drugs, like warfarin, diazepam, alprazolam, amitryptiline, clomipramine, clozapine, imipramine, mexiletine or thioridazine $(18,25,26)$.

Table 4. Bioequivalence (bioeq.) evaluation of the pharmacokinetic (PK) parameters corresponding to nebivolol (NEB) and 4-OH-nebivolol (4-OH-NEB; active metabolite), before and after pretreatment with fluvoxamine (50-100 $\mathrm{mg} / \mathrm{day}, 6$ days).

\begin{tabular}{llll}
\hline $\begin{array}{l}\text { Parent drug } \\
\text { /metabolite }\end{array}$ & PK parameter & $\mathbf{9 0} \% \mathbf{C I}^{*}$ & $\begin{array}{l}\text { Bioeq. } \\
\text { conclusion** }\end{array}$ \\
\hline \multirow{2}{*}{ NEB } & $\mathrm{C}_{\max }$ & $1.05-1.63$ & Bio-ineq \\
& $\mathrm{AUC}_{0-\mathrm{t}}$ & $1.27-1.82$ & Bio-ineq \\
& $\mathrm{AUC}_{0-\infty}$ & $1.25-1.85$ & Bio-ineq \\
\hline \multirow{2}{*}{$\mathbf{4 - O H - N E B}$} & $\mathrm{t}_{\max }$ & Friedman & Bio-eq \\
& $\mathrm{C}_{\max }$ & $1.26-1.61$ & Bio-ineq \\
& $\mathrm{AUC}_{0-\mathrm{t}}$ & $1.14-1.65$ & Bio-ineq \\
& $\mathrm{AUC}_{0-\infty}$ & $1.05-1.67$ & Bio-ineq \\
& $\mathrm{t}_{\max }$ & Friedman & Bio-eq \\
\hline $90 \%$ CI- $90 \%$ confidence intervals; **Bioequivalent if $90 \%$ CI: $0.8-1.25 ;$ Bio-ineq: Bio-inequivalent
\end{tabular}




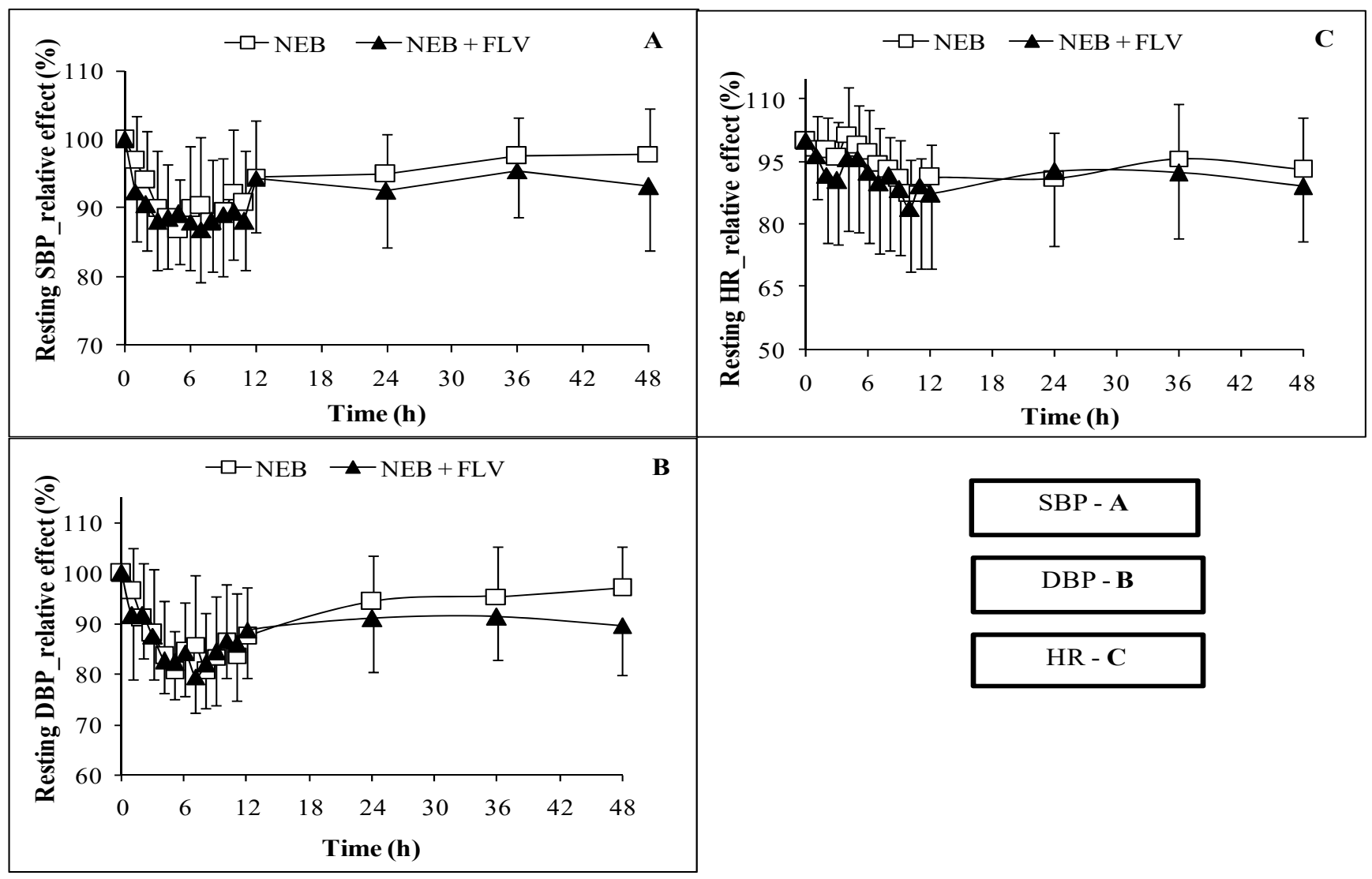

Figure 3. Changes in mean ( \pm standard deviation (SD)) systolic blood pressure (SBP-a), diastolic blood pressure (DBP-b) and heart rate (HR-c) measured at rest, following oral administration of single-dose $5 \mathrm{mg}$ nebivolol (NEB), before ( $\square$ ) and after fluvoxamine (FLV) pretreatment $(\boldsymbol{\Delta})$ for 6 days $(50-100 \mathrm{mg} /$ day), in 18 healthy volunteers (extensive metabolizers EMs).

As mentioned previously, in view of the fact that depression can often coexist with cardiovascular disease and its associated risk factors, including hypertension (27), the present study aimed to investigate whether fluvoxamine, a weak inhibitor of CYP2D6 (18), has an impact upon the pharmacokinetics of nebivolol, a cardiovascular drug that is a substrate of the same metabolic pathway. The potential pharmacokinetic drug interaction between nebivolol and fluvoxamine was investigated in volunteers considered to be EMs. All data obtained from subjects regarded as PMs were removed from the final report in order to avoid any interference with the outcome of the research. This resolution can be attributed to the fact that, according to the scientific literature, drug interactions do not occur in PMs as the activity of CYP2D6 is already compromised for this particular phenotype (28).

As reported in the study results, repeated doses of fluvoxamine influenced the pharmacokinetics of nebivolol administered as a single-5 $\mathrm{mg}$ dose and its active hydroxylated metabolite. Figure 1 revealed that the addition of fluvoxamine altered the mean plasma concentrations of the parent drug, respectively nebivolol plasma levels were increased during Test study period when the beta-blocker was associated with the enzymatic inhibitor. The plasmatic profile of 4-OH-nebivolol presented in Figure 2 exposed that the mean plasma concentrations of this active metabolite were also raised after the concomitant use of nebivolol and fluvoxamine. Furthermore, the pharmacokinetic analysis showed that fluvoxamine altered the pharmacokinetic parameters of nebivolol and its active metabolite, although the overall impact was rather small. For both analytes, significant differences between their pharmacokinetic profiles corresponding to each study period were observed for $\mathrm{C}_{\max }, \mathrm{AUC}_{0-\mathrm{t}}$ and $\mathrm{AUC}_{0-\infty}$, thus suggesting a potential presystemic drug interaction. The pharmacokinetic evaluation of nebivolol concluded that fluvoxamine was responsible for a 1.31-fold increase in $\mathrm{C}_{\max }$ and 1.59-fold increase in $\mathrm{AUC}_{0-\infty}$ 
(Table 2). Key pharmacokinetic parameters of 4$0 \mathrm{H}$-nebivolol were also influenced by this enzymatic inhibitor as a 1.41-fold and 1.44-fold increase in $\mathrm{C}_{\max }$ and $\mathrm{AUC}_{0-\infty}$ were observed when nebivolol was administered in combination with the antidepressant in comparison with the beta-blocker administered as monotherapy (Table 3). No significant changes were reported for the other parameters, respectively $t_{\max }, k_{\mathrm{el}}$ and $t_{1 / 2}$. These results demonstrated that an increased exposure to nebivolol and its active metabolite was observed after fluvoxamine concomitant intake, which provided evidence that a metabolic drug-drug interaction was present between the two drugs.

In comparison to other antidepressants, also known as CYP2D6 inhibitors, the present research revealed that the effect of fluvoxamine on the pharmacokinetics of nebivolol was smaller. Potent inhibitors of the same metabolic pathway, like fluoxetine (12), paroxetine (22) and bupropion (29) had a marked influence upon nebivolol pharmacokinetics and were responsible for an approximately 6-fold to 7-fold increase in $\mathrm{AUC}_{0-\infty}$. The association of the beta-blocker with bupropion and paroxetine also revealed a 4-fold and 5.7-fold increased exposure to 4-OH-nebivolol, the active hydroxylated metabolite $(22,29)$. On the other hand, pretreatment with duloxetine, a moderate CYP2D6 enzymatic inhibitor, had an impact on nebivolol pharmacokinetics, similar with the one attributed to fluvoxamine in this study. More precisely, in a clinical study that included healthy volunteers, duloxetine was responsible for a 1.19-fold and 1.42fold increase in the $\mathrm{AUC}_{0-\infty}$ values of nebivolol and its hydroxylated active metabolite (30), which suggests a similar inhibition potency of isoenzyme CYP2D6 for duloxetine and fluvoxamine. After evaluating the magnitude of all the aforementioned metabolism-mediated pharmacokinetic interactions involving nebivolol and antidepressants, fluvoxamine had a much smaller influence upon nebivolol pharmacokinetics compared to other antidepressants like bupropion, paroxetine or fluoxetine. Until now, very few studies have investigated the potential pharmacokinetic interaction between fluvoxamine and CYP2D6 substrates, all of which have revealed that this enzymatic inhibitor produced only small changes in the pharmacokinetics of desipramine, dextrometorphan or risperidone (31-33). The present study results also confirmed this hypothesis that only a modest impact on nebivolol exposure can be noticed after its association with fluvoxamine.
The bioequivalence evaluation of nebivolol and its hydroxylated metabolite between the two study periods revealed that the $90 \% \mathrm{CI}$ for the main pharmacokinetic parameters $\left(\mathrm{C}_{\max }, \mathrm{AUC}_{0-\mathrm{t}}, \mathrm{AUC}_{0-\infty}\right)$ were not in the acceptable limit range of bioequivalence $(0.8-1.25)$, thus hinting that the pharmacokinetic interaction may have clinical significance (Table 4). Pharmacological aspects should also be taken into account when evaluating the results of the present research. Considering that inflammation is involved in the pathogenesis of depression, as well as of cardiovascular disorders (34) and that this process can influence metabolizing enzymes, transporters, receptors and plasma proteins as a result of gene expression alterations (35), it can be presumed that response to pharmacotherapy may be altered in such a context. Pharmacological studies demonstrated that inflammation is associated with a reduced clearance of highly protein-bound and extensively metabolized drugs. Nonetheless, although increased plasma concentrations of propranolol were found using an animal model of inflammation, the beta-blocker displayed a decreased effectiveness that was finally attributed to reduced expression of $\beta 1$ receptor proteins $(35,36)$. However, nebivolol is a unique representative among the beta-blockers and unlike propranolol, both its action and disposition were unaffected by inflammation despite the reduced $\beta 1$-adrenoceptor levels (37). For this reason, the therapeutic response to nebivolol might not be influenced by the presence of inflammatory conditions like the ones depicted in the present study, but a more comprehensive investigation should be contemplated.

In order to obtain information concerning the clinical relevance of this pharmacokinetic interaction, a pharmacodynamic analysis which involved measurements of vital signs (SBP, DBP and HR) at rest was performed after nebivolol intake, in the presence and absence of fluvoxamine. The purpose of this assessment was to underline any potentiated effects induced by nebivolol and its active metabolite after their increased exposure as a result of the pharmacokinetic drug interaction. The 48hour time course of the antihypertensive effect induced by nebivolol revealed a similar profile irrespective of the coadministration of the enzymatic inhibitor. Likewise, the decrease in HR produced by the beta-blocker was also comparable (Figure 3). Hence, the graphical representation of the effect exerted by nebivolol upon SBP, DBP and HR during the two study periods (Test vs Reference) suggested that the combination of nebivolol and fluvoxamine did not enhance the BP- and HR-lowering effects of 
the beta-blocker. Also, no statistically significant differences were found between the mean pharmacodynamic parameters measured during Reference and Test period. Therefore, even if the present study demonstrated that fluvoxamine had an effect upon nebivolol pharmacokinetics, the pharmacodynamic evaluation suggests that this interaction may not have clinical consequences. A similar outcome was obtained in previous studies. Several clinical trials investigated not only whether a drug interaction does exist between enzymatic inhibitors like cimetidine, duloxetine, paroxetine or bupropion and nebivolol, but also whether clinical consequences are associated with these potential drug interactions. Although it was revealed that the aforementioned drugs determined an increased exposure to nebivolol, these drug combinations did not lead to greater reductions of the pharmacodynamic parameters (BP and HR) measured at rest or during exercise $(22,23,29,30)$. Additionally, no published case reports that describe side effects induced by nebivolol when coadministered with fluvoxamine were found in the medical literature.

An important aspect that has to be considered when analyzing the pharmacodynamic outcome is that the present research comprised healthy volunteers and not hypertensive patients. These healthy volunteers received a single-dose of nebivolol, which is different from the repeated administration regimen usually encountered in clinical practice. But, even if the study included only information related to nebivolol given as a singledose, the scientific literature offers strong evidence regarding its ability in influencing hemodynamic parameters such as BP and HR not only in hypertensive patients, but in healthy subjects as well. For example, a double-blind, placebo-controlled, crossover study that included male hypertensive patients revealed that, according to a 24 -h post-dose evaluation of BP and HR measured at rest and during submaximal exercise, nebivolol (5 $\mathrm{mg}$ and 10 $\mathrm{mg}$, once-daily), was indeed capable of reducing these parameters (38). Moreover, nebivolol (5 mg, once-daily) displayed a gradual onset of its BPlowering effects in patients with uncomplicated mild to moderate primary hypertension and it was also noted that a reduction in SBP was detected 5-6 h after the initial dose (39). A similar situation was encountered in studies in which this beta-blocker was administered in healthy subjects. According to Van Bortel et al, the beta-blocking effects of nebivolol were considered to be smaller after oral intake of a single dose $(5 \mathrm{mg})$ compared to the effects exerted after a 7-day course of therapy (40). Therefore, although with a lower potency, singledose nebivolol proved to be effective in reducing BP and HR in healthy subjects. In addition, a randomized, double-blind, placebo-controlled, crossover trial that investigated the beta-blocking effects of nebivolol, carvedilol and bisoprolol demonstrated that these agents were able to reduce SBP and HR at rest and during exercise at 3 and 24$\mathrm{h}$ following intake in healthy volunteers (41).

Thus, the present results can be viewed as useful preliminary clinical data. However, some important aspects regarding the pharmacodynamic effects of nebivolol have to be acknowledged if a conclusive decision regarding the absence or presence of any clinical consequences associated with this drug combination is to be made. For example, nebivolol has a maximal antihypertensive effect after 2 to 8 weeks of therapy (42). As for the heart rate, pronounced reductions were also evident only after weeks of continued therapy with this beta-blocker (43). Nonetheless, in comparison to atenolol, nebivolol was associated with a less marked bradycardic effect during the first 4 weeks of treatment in hypertensive patients. It was speculated that this characteristic can be attributed to the fact that the vasodilator effect that accompanies the betablocking action can attenuate the vagal tone (44). In addition, when investigating clinical aspects it is also important to analyze any data regarding the doseeffect relationship of a certain drug. In the case of nebivolol, according to a review that evaluated the BP lowering efficacy of beta-1 selective betablockers for primary hypertension, doses in the range of 1 to $40 \mathrm{mg}$ daily significantly reduced SBP and DBP in a total of 3209 hypertensive patients (12 randomized controlled trials). It was also stated that the maximum antihypertensive effect was seen at 5 $\mathrm{mg} /$ day and no dose-related effect was observed at doses $>5 \mathrm{mg} /$ day. These higher doses did not provide any additional BP lowering effects. The same source estimated that the BP lowering efficacy of this beta-blocker is $-8 /-6 \mathrm{mmHg}$ (45). Therefore, in order to simulate the therapeutic effect of nebivolol, the dose chosen to be administered throughout the study was $5 \mathrm{mg} /$ day. In light of all the aspects mentioned above, it should be noted that the extrapolation of the current results to hypertensive patients receiving a dosing regimen of repeated nebivolol and fluvoxamine administration can be viewed as debatable as it remains unknown whether the interaction might become clinically relevant when multiple-dose nebivolol is combined with multiple-dose fluvoxamine. A small amount of 
data can be found in the scientific literature regarding the correlation between plasma levels and clinical response to nebivolol, but according to Lindamood et al, clinically safe and well-tolerated levels of nebivolol alone were previously observed in PMs with a pharmacokinetic profile which included the following mean parameters: $\mathrm{AUC}_{0-\infty}$ $614 \mathrm{ng} * \mathrm{~h} / \mathrm{mL}$ and $\mathrm{C}_{\max } 9.2 \mathrm{ng} / \mathrm{mL}$ (12). In comparison to these values, the peak plasma levels and the $\mathrm{AUC}_{0-\infty}$ value obtained after nebivolol was administered with fluvoxamine were notably lower, which could explain the difficulty in revealing any clinical significance in the present research.

Therefore, for a definite conclusion, additional studies that include hypertensive patients and a repeated dosage regimen for nebivolol should be considered. When initiating further enquiries regarding the present pharmacokinetic interaction, another aspect that should be taken into account is the dosage regimen for fluvoxamine as one study revealed that a dose of $150 \mathrm{mg}$ /day produced a significant inhibition of CYP2D6 activity, which further supports the hypothesis that higher doses of this antidepressant could be responsible for relevant drug interactions (46).

\section{Limitations}

A major study limitation refers to the lack of any data regarding genotyping. Although a genotype analysis was not performed, the possibility of including subjects with a PM status was taken into consideration and addressed by conducting a phenotype analysis that included the investigation of the AUC metabolic ratio (nebivolol/4-OH-nebivolol) for each volunteer. Nonetheless, we acknowledge as another important limit of this research the fact that the phenotyping method adopted was not a validated one as adequate probe drugs such as debrisoquine, sparteine, metoprolol or dextromethorphan (47) were not used. However, considering that nebivolol is primarily metabolized by CYP2D6 (9), the analysis of AUC metabolic ratios (nebivolol/4-OH-nebivolol) was useful in distinguishing two different groups that were labeled as EMs and PMs .

Additionally, the lack of emphasis on the stereochemistry of nebivolol, as well as the absence of randomization could also be considered as flaws of the present research. An important question that can be raised is whether the pharmacokinetic changes suffered by a certain enantiomer of nebivolol after pretreatment with fluvoxamine could explain the clinical data obtained in the present research. This aspect should be contemplated as the enantiomers of nebivolol have different pharmacologic properties (6). However, a previous study that investigated the effects of cimetidine on the pharmacokinetics and pharmacodynamics of nebivolol in healthy volunteers reported that the pharmacokinetic parameters of both enantiomers plus their hydroxylated metabolites showed very similar variations when comparing nebivolol + placebo versus nebivolol + cimetidine. Thus, it was proved that a pharmacokinetic interaction between cimetidine and nebivolol does exist, but the enzymatic inhibitor did not have a greater impact on the pharmacokinetics of a certain enantiomer when compared with the other. The same study revealed no statistically significant effects of this drug combination on the resting vital signs and exercise data (23).

With regard to the pharmacodynamic analysis, the assessment of vital signs (BP and HR), in the absence/presence of drug interaction, only at rest, can be viewed as a study limitation. Taking into consideration that adrenergic activity is increased during exercise, we may assume that the impact of increased exposure of nebivolol upon BP and HR could be more visible when evaluated during exercise.

\section{CONCLUSIONS}

The experimental data proved that multiple-dose fluvoxamine influenced the pharmacokinetics of nebivolol and its active hydroxylated metabolite. This interaction had no significant consequences upon nebivolol pharmacodynamics, but, before drawing a definite conclusion regarding the potential clinical implications of this pharmacokinetic drug interaction, further investigations are required.

\section{ACKNOWLEDGEMENTS}

Financial disclosures: This work was supported by the National Research Council (CNCS) Romania project PN-II-ID-PCE-2011-3-0731. All authors are full-time employees of the University of Medicine and Pharmacy "Iuliu Hatieganu", Cluj-Napoca, Romania.

\section{CONFLICTS OF INTEREST}

There are no conflicts of interest to be declared.

\section{REFERENCES}

1. Gao Y, Vanhoutte PM. Nebivolol : An endothelium- 
friendly selective $\beta 1$-adrenoceptor blocker. J Cardiovasc Pharmacol. 2012;59(1):16-21. doi: 10.1097/FJC.0b013e3182073e27.

2. Baldwin CM, Keam SJ. Nebivolol: In the treatment of hypertension in the US. Am J Cardiovasc Drugs. 2009;9(4):253-60. doi: 10.2165/1120274000000000-00000.

3. Gupta S, Wright HM. Nebivolol: A highly selective beta1-adrenergic receptor blocker that causes vasodilation by increasing nitric oxide. Cardiovasc Ther. 2008;26(3):189-202. doi: 10.1111/j.17555922.2008.00054.x.

4. Wojciechowski D, Papademetriou V. Beta-blockers in the management of hypertension: focus on nebivolol. Expert Rev Cardiovasc Ther. 2008;6(4):471-9. doi: 10.1586/14779072.6.4.471.

5. Howlett JG. Nebivolol: Vasodilator properties and evidence for relevance in treatment of cardiovascular disease. Can J Cardiol. 2014;30(5 S). doi: 10.1016/j.cjca.2014.03.003.

6. Ignarro LJ. Different pharmacological properties of two enantiomers in a unique beta-blocker, nebivolol. Cardiovasc Ther. 2008;26(2):115-34. doi: 10.1111/j.1527-3466.2008.00044.x.

7. Toblli JE, Digennaro F, Fernando Giani J, Pablo Dominici F. Nebivolol: Impact on cardiac and endothelial function and clinical utility. Vasc Health Risk Manag. 2012;8(1):151-60. doi: 10.2147/VHRM.S20669.

8. Sanaee F, Valente Neves D, Lanchote VL, Jamali F. Pharmacokinetics of nebivolol in the rat: low oral absorption, loss in the gut and systemic stereoselectivity. Biopharm Drug Dispos. 2013;34(6):312-20. doi: 10.1002/bdd.1847.

9. De Boer RA, Voors AA, Van Veldhuisen DJ. Nebivolol : third-generation $\beta$-blockade. Expert Opin. Pharmacother. 2007;8(10):1539-1550. doi: 10.1517/14656566.8.10.1539.

10. Gray CL, Ndefo UA. Nebivolol: A new antihypertensive agent. Am J Heal Pharm. 2008;65(12):1125-33. doi: 10.2146/ajhp070459.

11. Cheng JWM. Nebivolol: a third-generation betablocker for hypertension. Clin Ther. 2009;31(3):44762. doi: 10.1016/j.clinthera.2009.03.007.

12. Lindamood C, Ortiz S, Shaw A, Rackley R, Gorski JC. Effects of commonly administered agents and genetics on nebivolol pharmacokinetics: drug-drug interaction studies. J Clin Pharmacol. 2011;51(4):575-85. doi: 10.1177/0091270010370846.

13. Fongemie J, Felix-Getzik E. A Review of nebivolol pharmacology and clinical evidence. Drugs. 2015;75(12):1349-71. doi: 10.1007/s40265-0150435-5.

14. Gielen W, Cleophas TJ, Agrawal R. Nebivolol: a review of its clinical and pharmacological characteristics. Int $\mathrm{J}$ Clin Pharmacol Ther. 2006;44(8):344-57. doi: 10.5414/CPP44344.

15. Mandrioli R, Mercolini L, A. Saracino M, A. Raggi M. Selective serotonin reuptake inhibitors (SSRIs):
Therapeutic drug monitoring and pharmacological interactions. Curr Med Chem. 2012;19:1846-63. doi: 10.2174/092986712800099749.

16. Altamura AC, Caldiroli A, Buoli M. Pharmacokinetic evaluation of fluvoxamine for the treatment of anxiety disorders. Expert Opin Drug Metab Toxicol. 2015;11(4):649-60. $10.1517 / 17425255.2015 .1021331$.

17. Hiemke C, Härtter S. Pharmacokinetics of selective serotonin reuptake inhibitors. Pharmacol Ther. 2000;85(1):11-28. doi:10.1016/S01637258(99)00048-0.

18. Spina E, de Leon J. Clinically relevant interactions between newer antidepressants and secondgeneration antipsychotics. Expert Opin Drug Metab Toxicol. 2014;10(5):721-46. doi: $10.1517 / 17425255.2014 .885504$.

19. Celano CM, Huffman JC. Depression and cardiac disease: A review. Cardiol Rev. 2011;19(3):130-42. doi: 10.1097/CRD.0b013e31820e8106.

20. Rutledge T, Reis VA, Linke SE, Greenberg BH, Mills PJ. Depression in heart failure. A meta-analytic review of prevalence, intervention effects, and associations with clinical outcomes. J Am Coll Cardiol. 2006;48(8):1527-37. doi:10.1016/j.jacc.2006.06.055.

21. Faul F, Erdfelder E, Lang AG, Buchner A. G*Power 3: A flexible statistical power analysis program for the social, behavioral, and biomedical sciences. Behav Res Methods. 2007;39(2):175-91. doi: 10.3758/bf03193146.

22. Briciu C, Neag M, Muntean D, Vlase L, Bocsan C, Buzoianu A, et al. A pharmacokinetic drug interaction study between nebivolol and paroxetine in healthy volunteers. J Clin Pharm Ther. 2014;39(5):535-40. doi: 10.1111/jcpt.12180.

23. Kamali F, Howes A, Thomas SH, Ford GA, Snoeck E. A pharmacokinetic and pharmacodynamic interaction study between nebivolol and the H2receptor antagonists cimetidine and ranitidine. $\mathrm{Br} \mathrm{J}$ Clin Pharmacol. 1997;43(2):201-4. doi: 10.1046/j.1365-2125.1997.54212.x

24. Lane RM. Pharmacokinetic drug interaction potential of selective serotonin reuptake inhibitors. Int Clin Psychopharmacol. 1996;11(Suppl 5):31-61.

25. Baumann P. Pharmacokinetic-pharmacodynamic relationship of the selective serotonin reuptake inhibitors. Clin Pharmacokinet. 1996;31(6):444-69. doi:10.2165/00003088-199631060-00004.

26. Hemeryck A, Belpaire FM. Selective serotonin reuptake inhibitors and cytochrome P-450 mediated drug-drug interactions : An update. 2002;3(1):13-37. doi: $10.2174 / 1389200023338017$.

27. Elderon L, Whooley MA. Depression and cardiovascular disease. Prog Cardiovasc Dis. 2013;55(6):511-23. doi: 10.1016/j.pcad.2013.03.010.

28. Teh LK, Bertilsson L. Pharmacogenomics of CYP2D6: molecular genetics, interethnic differences and clinical importance. Drug Metab Pharmacokinet. 
2012;27(1):55-67. doi: 10.2133/dmpk.DMPK-11$\mathrm{RV}-121$.

29. Gheldiu A-M, Popa A, Neag M, Muntean D, Bocsan C, Buzoianu A, et al. Assessment of a potential pharmacokinetic interaction between nebivolol and bupropion in healthy volunteers. Pharmacology. 2016;98(3-4):190-8. doi: 10.1159/000447266.

30. Briciu C, Neag M, Muntean D, Vlase L, Bocsan C, Buzoianu A, et al. A pharmacokinetic drug interaction study between nebivolol and duloxetine. Exp Clin Cardiol. 2014;20(1):1374-79.

31. D'Arrigo C, Migliardi G, Santoro V, Morgante L, Muscatello MR, Ancione M, et al. Effect of fluvoxamine on plasma risperidone concentrations in patients with schizophrenia. Pharmacol Res. 2005;52(6):497-501. doi: 10.1016/j.phrs.2005.09.005.

32. Alfaro CL, Lam YW, Simpson J, Ereshefsky L. CYP2D6 inhibition by fluoxetine, paroxetine, sertraline, and venlafaxine in a crossover study: intraindividual variability and plasma concentration correlations. J Clin Pharmacol. 2000;40(1):58-66. doi: 10.1177/009127000004000108.

33. Spina E, Pollicino AM, Avenoso A, Campo GM, Perucca E, Caputi AP. Effect of fluvoxamine on the pharmacokinetics of imipramine and desipramine in healthy subjects. Ther Drug Monit. 1993;15(3):243-6.

34. Kulmatycki KM, Jamali F. Drug disease interactions: role of inflammatory mediators in depression and variability in antidepressant drug response. J Pharm Pharm Sci. 2006;9(3):292-306.

35. Hanafy S, El-Kadi AOS, Jamali F. Effect of inflammation on molecular targets and drug transporters. J Pharm Pharm Sci. 2012;15(3):361-75. doi: $10.18433 / \mathrm{J} 30300$.

36. Kulmatycki KM, Jamali F. Drug disease interactions: role of inflammatory mediators in disease and variability in drug response. J Pharm Pharm Sci. 2005;8(3):602-25.

37. Sanaee F, Jamali F. Action and disposition of the $\beta 3$ agonist nebivolol in the presence of inflammation; an alternative to conventional $\beta 1$-blockers. Curr Pharm Des. 2014;20(9):1311-7. doi: $10.2174 / 13816128113199990550$.

38. Simon G, Johnson ML. Comparison of antihypertensive and beta 1-adrenoceptor antagonist effect of nebivolol and atenolol in essential hypertension. Clin Exp Hypertens. 1993;15(3):501-9. doi: 10.3109/10641969309041625.

39. Himmelmann A, Hedner T, Snoeck E, Lundgren B, Hedner J. Haemodynamic effects and pharmacokinetics of oral $\mathrm{d}$ - and 1-nebivolol in hypertensive patients. Eur J Clin Pharmacol. 1996;51(3-4):259-64. doi: 10.1007/s002280050194.

40. Van Bortel LM, de Hoon JN, Kool MJ, Wijnen JA, Vertommen CI, Van Nueten LG. Pharmacological properties of nebivolol in man. Eur J Clin Pharmacol. 1997;51(5):379-84. doi: 10.1007/s002280050217.

41. Stoschitzky K, Stoschitzky G, Brussee H, Bonell C, Dobnig H. Comparing beta-blocking effects of bisoprolol, carvedilol and nebivolol. Cardiology. 2006;106(4):199-206. doi: 10.1159/000093060.

42. Kumar D, Loc B. Nebivolol a novel somewhat different beta adrenergic receptor blocker. J Drug Deliv Ther. 2013;3(6):185-91.

43. McNeely W, Goa KL. Nebivolol in the management of essential hypertension: a review. Drugs. 1999;57(4):633-51. doi: 10.2165/00003495199957040-00011.

44. Grassi G, Trevano FQ, Facchini A, Toutouzas T, Chanu B, Mancia G. Efficacy and tolerability profile of nebivolol vs atenolol in mild-to-moderate essential hypertension: results of a double-blind randomized multicentre trial. Blood Press Suppl. 2003;12:35-40. doi: 10.1080/08038020310023271.

45. Wong GW, Boyda HN, Wright JM. Blood pressure lowering efficacy of beta-1 selective beta blockers for primary hypertension. Cochrane Database Syst Rev. 2016;10;3:CD007451. doi: 10.1002/14651858.CD007451.pub2.

46. Kashuba ADM, Nafziger AN, Kearns GL, Leeder JS, Gotschall R, Rocci ML, et al. Effect of fluvoxamine therapy on the activities of CYP1A2, CYP2D6, and CYP3A as determined by phenotyping. Clin Pharmacol Ther. 1998;64(3):257-68. doi: 10.1016/S0009-9236(98)90174-6.

47. Frank D, Jaehde U, Fuhr U. Evaluation of probe drugs and pharmacokinetic metrics for CYP2D6 phenotyping. Eur J Clin Pharmacol. 2007;63(4):32133.doi: 10.1007/s00228-006-0250-8. 